\title{
Guidelines for the management of human brucellosis in the State of Paraná, Brazil
}

\author{
Felipe Francisco Tuon ${ }^{[1],[2], ~ N a t a c h a ~ C e r c h i a r i ~}{ }^{[1]}$, Juliana Clélia Cequinel ${ }^{[3]}$, \\ Elizabeth El Hajjar Droppa ${ }^{[4]}$, Suzana Dal Ri Moreira ${ }^{[5]}$, Tânia Portella Costa ${ }^{[3]}$, \\ Amanda de Paula Boni Navarro ${ }^{[6]}$, Antonieta Milleo Handar ${ }^{[6]}$, Margely Nunes de Souza ${ }^{[7]}$ \\ and Brucellosis Workgroup*
}

\begin{abstract}
[1]. Divisão de Doenças Infecciosas, Hospital de Clínicas, Universidade Federal do Paraná, Curitiba, PR, Brasil. [2]. Escola de Medicina, Pontifícia Universidade Católica do Paraná, Curitiba, PR, Brasil. [3]. Divisão de Vigilância de Zoonoses e Intoxicação, Centro Estadual de Vigilância Ambiental, Secretaria de Estado da Saúde do Paraná, Curitiba, PR, Brasil. [4]. Laboratório Central do Estado do Paraná, Secretaria de Estado da Saúde do Paraná, Curitiba, PR, Brasil. [5]. Centro de Informações Estratégicas em Vigilância em Saúde, Secretaria de Estado da Saúde do Paraná, Curitiba, PR, Brasil.

[6]. Centro de Saúde do Trabalhador, Secretaria de Estado da Saúde do Paraná, Curitiba, PR, Brasil. [7]. Centro de Medicamentos do Paraná, Secretaria de Estado da Saúde do Paraná, Curitiba, PR, Brasil.
\end{abstract}

\begin{abstract}
Human brucellosis is a re-emerging disease with the potential for bioterrorism. The number of cases in Brazil has increased; however, the ideal management has not been established. These guidelines are intended for use by clinicians and other health-care workers providing medical care for patients with suspected brucellosis in the State of Paraná. We included a brief description of the epidemiology, clinical presentation, diagnosis, prevention of exposure, prevention of disease by chemoprophylaxis, treatment of disease, monitoring of adverse effects during treatment, management of treatment failure and relapse cases.
\end{abstract}

Keywords: Brucella. Brucellosis. Guidelines. Humans.

\section{INTRODUCTION}

Brucellosis is a zoonotic disease caused by bacteria of the genus Brucella spp., found in different regions of the world. Human brucellosis causes serious health problems and economic $\operatorname{losses}^{1}$. It is considered a re-emerging disease and is a potential agent for bioterrorism ${ }^{2}$. It is common in the Middle East, Asia, Africa, South and Central America, the Mediterranean, and the Caribbean ${ }^{1}$. Currently, according to World Health Organization (WHO), it is believed that, even in developed countries, the true incidence of brucellosis may be at least five times higher than that officially reported, as a result of the underdiagnosed and underreported cases $^{3}$.

Brucellosis is a disease with a large spectrum of clinical presentations ${ }^{4}$. It can cause systemic symptoms or mild, localized to severe forms of disease, like meningitis and endocarditis. Human brucellosis can be characterized by fever, accompanied by other unspecific signs and symptoms such as asthenia, night sweats, anorexia, myalgia, back pain, and arthralgia. The common route of transmission is through direct contact with infected animals and their secretions, or by consumption of unpasteurized milk and/or dairy products 5 .

Brucellosis has emerged as a prevalent zoonotic disease thus becoming a public health concern in the State of Paraná, in Brazil. The increased number of human cases and accidental exposures to Brucella spp. highlight the need for guidelines that detail definitions, diagnosis, and treatment for those diagnosed with brucellosis, and the prophylaxis and management for pre and post exposure.

\section{METHODS}

\section{Group}

A Working Group was established as a resolution of the State of Paraná, Brazil (SESA 042/2015) to restructure and upgrade the State Protocol on Human Brucellosis. We convened a panel of 20 experts, including specialists in infectious diseases, biologists, veterinary specialists, laboratory specialists, epidemiologists, and pharmacists from State of Paraná, Brazil.

\footnotetext{
*Workgroup of Brucellosis of State of Paraná, Brazil: Célia Fagundes da Cruz (Laboratório Central do Estado do Paraná), Fabiana Menoncin Souza (Centro de Medicamentos do Paraná), Ivana Lucia Belmonte (Centro Estadual de Vigilância Ambiental), Karina Ruaro de Paula (Centro Estadual de Vigilância Sanitária/Divisão de Vigilância Sanitária de Alimentos), Laurina Setsuko Tanabe (Centro de Informações Estratégicas e Respostas em Vigilância em Saúde/Divisão de Unidade de Informação), Márcia Zinelli da Silveira (Centro Estadual de Vigilância Ambiental), Maria Carolina Lobo da Silva Leal (Secretaria de Estado da Saúde do Paraná), Mariza Koloda (Agência de Defesa Agropecuária do Paraná), Nelson Ricetti de Nazareno (Centro Estadual de Epidemiologia/Divisão de Informações Epidemiológicas), Pedro Paulo Pedroso (Secretaria de Estado da Saúde do Paraná), Yumie Murakami (Secretaria de Estado da Saúde do Paraná). 


\section{Literature review and analysis}

The panel members defined questions to be answered during the management of patients with brucellosis, which included: 1) epidemiological definitions of cases; 2) the most common signs and symptoms; 3) diagnostic tests and interpretation; 4) the treatment and follow-up; 5) definition of therapy failure and cure. The panel members also defined questions to be answered about the management of human exposure to Brucella in the following conditions: 1) vaccine exposition; 2) laboratorial; 3) patient contact; 4) workplace with infected animals with brucellosis.

Literature pertinent to each of the questions was reviewed using PubMed, Cochrane Library, Google Scholar, references in published articles or textbooks, official websites, and textbooks. There were no language limitations. The period for the literature search was not fixed, thus, any date could be consulted. Some keywords were used for the literature review however; a systematic search was not conducted. The following keywords were used in the main search: Brucella OR brucellosis, associated or not with other co-related words such as diagnosis, laboratory, tests, treatment, therapy, prevention, prophylaxis, vaccine accident, RB51, contamination, transmission, and case definition.

\section{Evidence level}

The definitions on the strength and quality of the recommendations were the same as those used by the European Society of Clinical Microbiology and Infectious Diseases (ESCMID) ${ }^{6}$. The strength of these recommendations was defined as follows: A, strongly recommended; B, moderately recommended; $\mathrm{C}$, marginally recommended; $\mathrm{D}$, not recommended. The quality of the evidence was defined as follows: level I, at least one properly designed randomized controlled trial; level II, well designed clinical trial without randomization, case-control, or cohort studies; or level III, opinion of respected authorities, case reports, and clinical experience. These recommendations were used for therapy and prophylaxis.

\section{Etiology and animal reservoir}

Brucella are Gram-negative coccobacilli, which are nonencapsulated, non-mobile, and which grow slowly in cultured media $^{5}$. Brucella are obligate aerobe and do not ferment carbohydrates. Some strains require carbon dioxide to grow. The colonies are smooth (translucent and homogeneous) or rough (opaque, granular, or sticky). Due to its characteristics, Brucella spp. cultivation requires biosafety laboratory level three; the isolated is classified as category A as a potential agent for the use of biological weapons by the Centers for Disease Control and Prevention (CDC) in the United States ${ }^{7}$. There are several species of Brucella, most of which are specific to some animals.

\section{Transmission}

General aspects: Brucella can be transmitted by contact with animal tissues, blood, urine, vaginal secretions, aborted fetuses, and especially placentae (i.e. ingestion of raw milk or unpasteurized milk products from infected animals). In the latter case, Brucella can survive for two weeks and up to six months in the product ${ }^{8}$. Meat is not a common means of transmission, since the numbers of bacteria are low and the consumption of raw meat is unusual. Brucella can be transmitted through the air to the animals in corrals and stables, and people in laboratories, farms, and slaughterhouses. Many cases occur through accidental selfinoculation of veterinary vaccines. Taking these aspects into consideration, we defined high risk groups for human brucellosis as follows: those working with live and/or slaughtered animals (i.e. animal handlers, veterinarians and their assistants, ranchers, butchers, and others); those working with refrigerators in slaughterhouses and/or abattoirs (i.e. those handling meat products, butchers during the slaughter and handling of meat products) ${ }^{2}$; workers dealing with milk, manufacturing dairy products, or related activities; those administering vaccinations who are prone to accidents (i.e. dermal inoculation during application of animal vaccines or contact of liquid vaccines in mucous); those working in laboratories who are prone to accidents (i.e. handling bacterial cultures, sniffing cultures or aerosols, direct contact with skin and conjunctiva); health professionals who handle biological material ${ }^{9,10}$.

Other forms of human transmission are possible, such as ingestion of contaminated vegetables by feces and/or urine of infected animals. Transmission between humans is rare, but it is possible via sexual and intrauterine transmission, breastfeeding, and organ and blood transfusions.

Vaccine exposure: The B19 vaccine is the vaccine of choice used in Brazil; it is included in the National Program of Control and Eradication of Brucellosis and Animal Tuberculosis. This vaccine is used only in female animals because it can cause orchitis. The B19 vaccine is produced using a strain of Brucella abortus which induces antibody production against the specific lipopolysaccharides (LPS $)^{11}$. This vaccine can infect humans because it is produced with live strains of Brucella. The vaccine RB 51 is produced with a different strain of $B$. abortus, following successive passages in medium containing sub-inhibitory concentrations of rifampin. As with the B19 vaccine, the RB 51 vaccination can infect humans, but with a strain resistant to rifampin.

\section{Clinical findings}

Brucellosis can be classified as acute or chronic, for didactical reasons. In cases of acute brucellosis, the symptoms are non-specific, such as fever, malaise, chills, weight loss, and arthralgia. The evolution of the disease can lead to spontaneous cure or it can progress into a chronic form. Chronic brucellosis can be localized in any organ, such as the central nervous system, and can lead to endocarditis, osteomyelitis, orchitis, and other conditions. The symptoms of acute and chronic forms can be unspecific, therefore, epidemiological data are very important $t^{12,13}$. The frequencies of the most common sign/ symptoms of brucellosis in adults and children are detailed in Table 1.

After an incubation period of 2 to 3 weeks, the first symptoms are fever, weight loss, myalgia, and profuse sudoresis. The characteristics of the fever are very variable, however, in some patients the fever is similar to that associated with tuberculosis, as it is accompanied with nocturne sudoresis. Arthralgia, 
TABLE 1

Frequency of signs and symptoms of brucellosis among adults and children.

\begin{tabular}{|c|c|c|}
\hline Signs and symptoms & Adults (\%) & Children (\%) \\
\hline Fever & 73.0 & 82.0 \\
\hline Arthralgia & 71.0 & 65.0 \\
\hline Myalgia & 56.0 & 18.0 \\
\hline Back pain & 49.0 & 10.0 \\
\hline Chills & 47.0 & 18.0 \\
\hline Headache & 34.0 & 9.0 \\
\hline Fatigue & 33.0 & 19.0 \\
\hline Splenomegaly & 24.0 & 31.0 \\
\hline Hepatomegaly & 22.0 & 27.0 \\
\hline Nausea/vomits & 16.0 & 0.0 \\
\hline Arthritis & 13.0 & 41.0 \\
\hline Spondylodiscitis & 12.0 & 18.0 \\
\hline Orchiepididymitis & 10.0 & 10.0 \\
\hline Neurologic symptoms & 5.0 & 2.0 \\
\hline Cutaneous lesions & 4.0 & 5.0 \\
\hline
\end{tabular}

myalgia, and headache are algic symptoms that occur during the initial stage. Later in brucellosis progression, the pain is less severe, but there is an increase in chronic fatigue. Gastrointestinal symptoms are also common, such as nausea, vomiting, abdominal pain, and diarrhea. Lymphadenopathy, hepatomegaly, and splenomegaly are also symptoms found in some patients ${ }^{5}$.

Osteomyelitis and arthritis are commonly associated with human brucellosis; they occur in $40 \%$ of brucellosis patients ${ }^{4}$. The most classic finding is sacroiliitis, which results in lower back pain associated with fever. Endocarditis and neurobrucellosis occur in cases with severe forms of brucellosis. Neurobrucellosis can be a large spectrum disease, associated with symptoms of depression, meningitis, or encephalitis ${ }^{14}$, and in some cases cranial nerve palsy. Brucellosis can lead to the compromise of the peripheral nervous system, leading to radiculopathy ${ }^{14}$. Lung diseases are common after inhalation of aerosols with Brucella ${ }^{15}$. The inhalation occurs during manipulation of cultures or clinical samples containing bacteria. Interstitial pneumonitis, pneumonia, bronchopneumonia, pleural effusion, and lung nodules are conditions related to Brucella infection $^{15}$.
Brucella infection during pregnancy is associated with abortion and intrauterine disease for the fetus. It is important to remember that Brucella can be transmitted by breast-feeding, thus exposed/infected mothers should take caution with their newborns. Neonatal Brucella infection is associated with bacteremia and acute diseases associated with organ dysfunction and respiratory distress ${ }^{16,17}$. Cutaneous lesions have been reported in patients with Brucella infection, as nodules, papules, erythema, and petechial. Although rare, ocular lesions have been reported in patients with brucellosis. Uveitis is the most common manifestation and may present as chronic iridocyclitis or optic neuritis. Brucella has been isolated from the human eye structures, however, many of these lesions have been considered late complications, possibly immunologically mediated ${ }^{18}$.

\section{Case definitions}

Suspect case: A patient with acute or insidious disease characterized by fever and one or more of the following signs or symptoms: night sweats, arthralgia, headache, fatigue, anorexia, myalgia, arthritis/spondylitis, meningitis, focal or organ involvement (endocarditis, orchitis/epididymitis, hepatomegaly 
and splenomegaly). Additionally, the patient had to have a suggestive epidemiological history of contact with contaminated animal products, occupational exposure, or handling animals affected by brucellosis.

Confirmed case: A suspected case, with coinciding laboratory confirmation of brucellosis.

Discarded case: A suspect case with laboratory findings negative for brucellosis and/or a diagnosis confirmed for another disease.

\section{Laboratory diagnosis}

The diagnostic tests for brucellosis can be direct or indirect. Direct methods include isolation and identification of Brucella, immunohistochemistry and detection of nucleic acids using polymerase chain reaction (PCR). Indirect tests detect antiBrucella antibodies which can be positive in patients with a history of brucellosis. The serological response to infection by Brucella is influenced by many factors such as the incubation period of the disease, which is highly variable and during which the serology may be negative. However, current serological tests have an accuracy of $95 \%$.

Culture is the gold standard method for the diagnosis of human brucellosis. Blood cultures using automated systems, have a high positivity rate; most of them show positive results within 7 days. It is not necessary incubate bottles longer than 7 days. Due to the high risk of laboratory-acquired infection, molecular methods for the speciation and subtyping of Brucella isolates have replaced the conventional method ${ }^{19}$.

The current working group recommends laboratory tests for suspected cases and serology and molecular tests for the diagnosis of brucellosis. Laboratory tests require $2 \mathrm{~mL}$ of serum and $3-5 \mathrm{~mL}$ of blood to be collected in serum and EDTA (ethylenediaminetetraacetic acid) tubes, respectively. The serum should be stored in specific tube between 2 and $8^{\circ} \mathrm{C}$ for 72 hours. After this period, the sample should be stored at $-20^{\circ} \mathrm{C}$. The blood should be stored in EDTA tubes between 2 and $8{ }^{\circ} \mathrm{C}$ for 72 hours; the blood samples cannot be frozen.

The methods chosen for laboratory diagnosis of brucellosis by this working group were Rose Bengal (serum agglutination), enzyme immunoassay (EIA) IgG and IgM, and real time PCR. The Rose Bengal test has high sensitivity and specificity, but positive results can occur in asymptomatic patients after exposure to Brucella or vaccination ${ }^{20}$. Real-time PCR was considered as the gold-standard method for diagnosis because Brucella can only be cultured in laboratories with at least a biosafety level for three ${ }^{21,22}$.

Symptomatic patients (symptoms suggesting brucellosis) with positive a PCR or a reagent EIA IgM should receive treatment, after excluding other potential causes of the symptoms. Patients with a reagent EIA IgG and a negative PCR and EIA IgM should not receive treatment; signs and symptoms should be evaluated or the patient should be referred to specialist for follow up. Patients with a positive isolated Rose Bengal should not receive treatment; the other serum tests (EIA IgG and IgM) should be repeated and the patient should be referred for to a specialist if the signs and symptoms are suggestive of brucellosis (Figure 1).

\section{Treatment}

Tetracycline (tetracycline, doxycycline and minocycline), aminoglycosides (amikacin, gentamycin, and streptomycin), quinolones (ciprofloxacin), rifampin, ceftriaxone, and sulphamethoxazole/trimethoprim are drugs active against

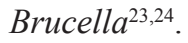

Monotherapy is inferior to combined therapy for the treatment of brucellosis. Therefore, at least two drugs should be used for the treatment of brucellosis (AI). Thirteen studies

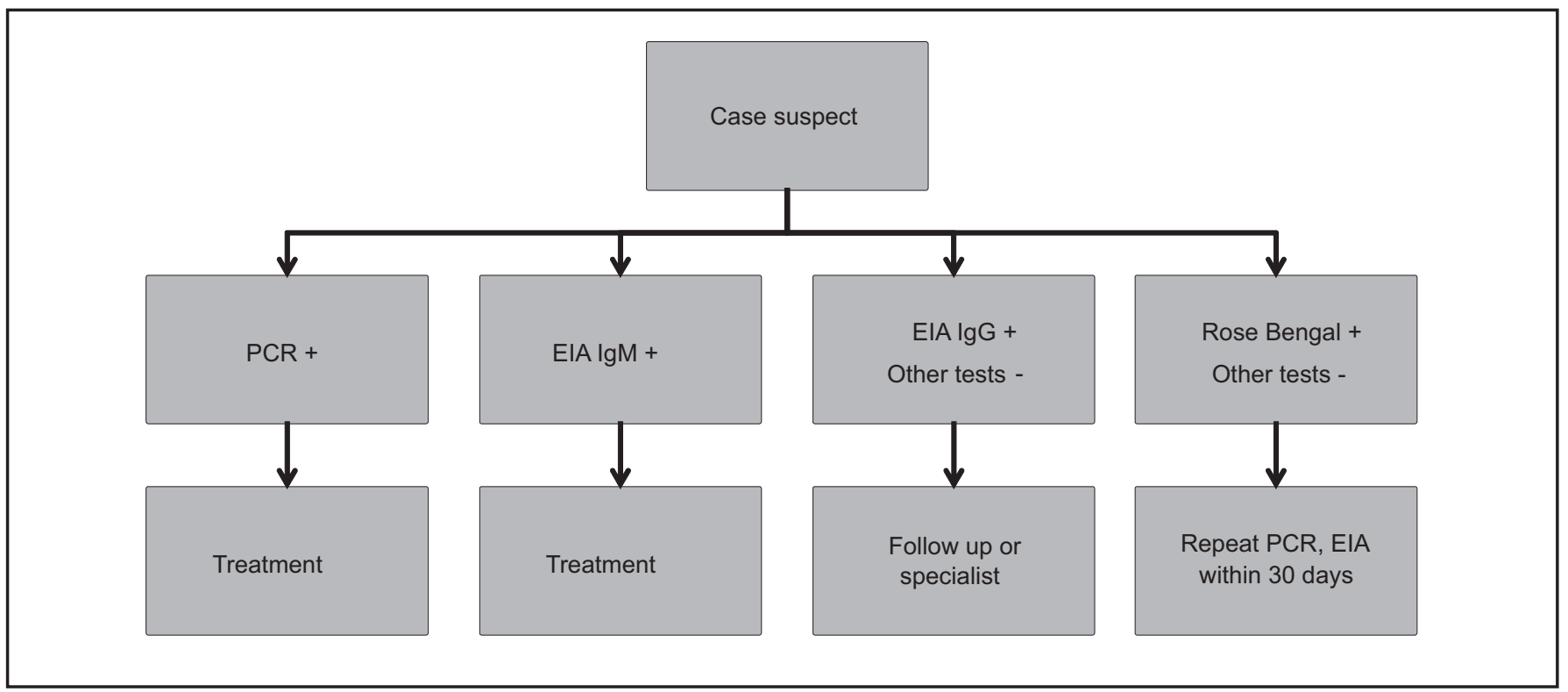

FIGURE 1 - Management of patients with brucellosis (suggestive symptoms) based on laboratory findings. Treatment should be initiated after excluding other potential causes of disease. PCR: polymerase chain reaction; EIA: enzyme immunoassay; IgM: immunoglobulin M; IgG: immunoglobulin G. 
comparing tetracycline associated with rifampin and tetracycline associated with gentamycin showed superiority of treatment with aminoglycosides (AII) ${ }^{25}$. These findings were confirmed by two more systematic reviews ${ }^{26,27}$. In the same year, a systematic review published by the Cochrane Group suggested the same treatment combination, however, with streptomycin as the aminoglycoside of choice ${ }^{28}$.

All aminoglycosides have presented similar efficacy in the treatment of brucellosis; however gentamycin had fewer side effects than streptomycin (BII). Unfortunately, there are very few studies that have investigated amikacin. In various studies, doxycycline was not inferior to tetracycline, however doxycycline had fewer side effects and can be used twice daily (BII). Treatment of brucellosis using quinolones was inferior to other drugs, and thus should not be used as the first line therapy (CII). Rifampin is another drug that can be considered as a substitution for doxycycline or aminoglycosides. However, the use of rifampin should be restricted because human brucellosis has similar symptoms with tuberculosis, and monotherapy with rifampin can lead to the selection of resistant $M$. tuberculosis strains. The treatment of choice for each site of infection with brucellosis is detailed in the Table 2.

\section{Follow up}

For patients with brucellosis, clinical and laboratory follow up is required for 2 years. The timeline suggested for follow-up is detailed in Table 3.

\section{Outcomes}

Failure was defined as the presence of signs and symptoms after 4 weeks of treatment and relapse was the return of signs and symptoms of brucellosis ${ }^{29}$. Patients were defined as cured if they did not meet the definitions of failure or relapse.

\section{Surveillance}

Suspect cases: Patients with compatible symptoms of brucellosis and with a suggestive epidemiological history (contact with contaminated material or ingestion of raw milk and dairy products from infected animals) should undergo clinical and laboratory evaluation. Establishment of the epidemiologic link is imperative to determine contacts with the same form of exposure in need of screening, particularly those with compatible symptoms. The suspected case must be notified using the individual notification form (ICD-10: A23) and must be reported to the Regional Health Division of the State of Paraná. If there is any doubt as to the clinical management of a case, interpretation of diagnostic results, treatment or follow-up, a reference physician for brucellosis is available at the Clinical Hospital of the Federal University of Paraná (HC/UFPR), through the Telehealth System platform. Samples for laboratory diagnosis should be directed to reference laboratories in the state (LACEN/PR), according to the guidelines for Collection and Shipping Biological Samples to Lacen / PR, available at www.lacen.saude.pr.gov.br.

Exposure to animals with brucellosis: The Agricultural Protection Agency of Paraná (ADAPAR) must officially notify the Municipal Health Department after the diagnosis of a case of animal brucellosis. The Health Surveillance system should then trigger investigation of exposed persons. An active search is conducted looking for all individuals exposed via direct contact (animal handling and their secretions i.e. tissue, blood, urine, vaginal secretions, aborted fetuses, and placenta) or indirect contact (milk intake or derived from animals affected by brucellosis). Collection of samples for diagnosis is indicated only for those exposed individuals with signs or symptoms of human brucellosis (suspect case definition). Individuals with suspected disease must be evaluated by a local health unit.

TABLE 2

Treatment of choice for patients with brucellosis according to age, weight and site.

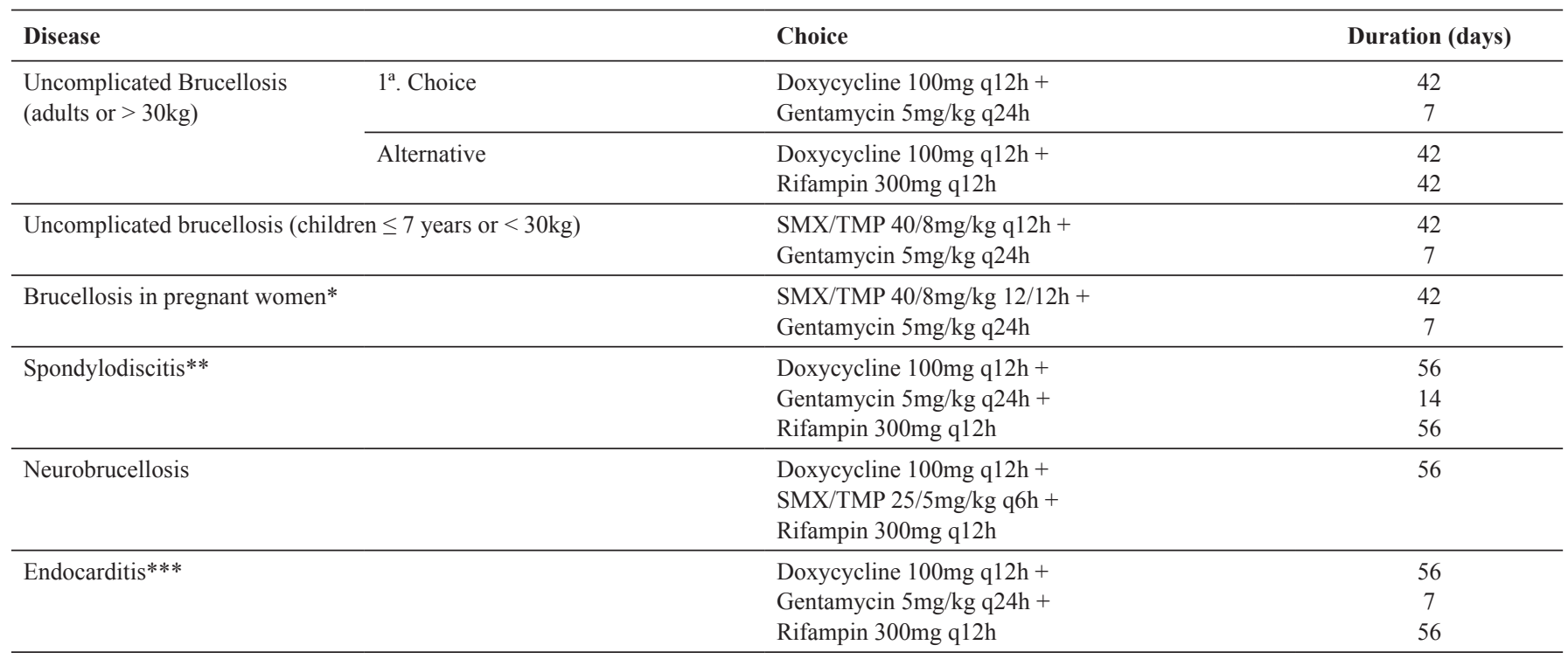

SMX: sulfamethoxazole; TMP: trimethoprim. *Avoid in the last 4 weeks (change to rifampin). ${ }^{*}$ The treatment can be longer in those with chronic infection, in general, symptoms for more than 6 weeks. ***Indicative of surgery 
TABLE 3

Clinical and laboratory follow up of patients with brucellosis.

\begin{tabular}{ll}
\hline Time & Follow up \\
\hline Week 1 & Evaluate drug adhesion \\
Week 2 & Clinical evaluation, hemogram, creatinine, urea, liver enzymes, erythrocyte sedimentation rate, reactive C protein \\
Week 4 & Clinical evaluation \\
Week 8 & Clinical evaluation, hemogram, creatinine, urea, liver enzymes, erythrocyte sedimentation rate, reactive C protein \\
Week 12 & Clinical evaluation, hemogram, creatinine, urea, liver enzymes, erythrocyte sedimentation rate, reactive C protein \\
Week 24 & Clinical evaluation, hemogram, erythrocyte sedimentation rate, reactive C protein \\
Month 12 & Clinical evaluation \\
Month 18 & Clinical evaluation \\
Month 24 & Clinical evaluation \\
\hline
\end{tabular}

TABLE 4

Definition of risk levels for post-exposure prophylaxis (adapted from the Center for Disease Control and Prevention, Griffith, 2008).

\begin{tabular}{llll}
\hline Risk level & Person at risk & Risk definition & PEP \\
\hline High & Individual & Sniffed or opened the culture plate using a maximum of BSL-2 precautions & Yes \\
\cline { 2 - 5 } & $\begin{array}{l}\text { Person performing activity } \\
\text { and any person within a } \\
\text { 5ft. radius }\end{array}$ & $\begin{array}{l}\text { Work with a Brucella isolate, sniffed or opened the culture plate, mouth pipetted } \\
\text { specimen material, worked in a Class II biosafety cabinet or on open bench with- } \\
\text { out using BSL-3 precautions }\end{array}$ & Yes \\
\cline { 2 - 5 } & $\begin{array}{l}\text { All persons present in } \\
\text { laboratory room }\end{array}$ & Occurrence of widespread aerosol generating procedures* & Yes \\
& $\begin{array}{l}\text { All persons present in } \\
\text { laboratory room at distance } \\
\text { greater than 5ft. from } \\
\text { activity }\end{array}$ & $\begin{array}{l}\text { Present in the lab at the time of manipulation of a Brucella isolate on an open } \\
\text { bench, but who do not have high risk exposures as defined above }\end{array}$ & May consider \\
& $\begin{array}{l}\text { All persons present in } \\
\text { laboratory room }\end{array}$ & $\begin{array}{l}\text { Handling and testing of a Brucella isolate in a Class II biosafety cabinet using } \\
\text { BSL-3 precautions }\end{array}$ \\
\hline None & None & \\
\hline
\end{tabular}

PEP: post-exposure prophylaxis; BSL: biosafety level. *Centrifuging without sealed carriers, vortexing, sonicating, accidents resulting in spillage or splashes (i.e. breakage of tube containing specimen).

Asymptomatic individuals must be advised about the disease and possible signs and symptoms.

Exposure to vaccine: For all accidents involving vaccinations, regardless of the vaccine, immediate post-exposure prophylaxis (PEP) should be provided to the exposed individual. The drug of choice for PEP was doxycycline, which should be administered in $100 \mathrm{mg}$ doses every 12 hours for 42 days.

It is important to promote health education and guidance on the use of Personal Protective Equipment (PPE) to avoid reoccurring accidents.

Exposure to laboratory material: All individuals' exposed to contaminated materials must be evaluated for the risk of exposure. The risk is stratified as high, low, or no risk. PEP is indicated for high and low risk exposures. The drug of choice for PEP was doxycycline, which should be administered in $100 \mathrm{mg}$ doses every 12 hours for 21 days. Exposed individual are followed up for 6 months with sequential serologic testing $(0,6,12,18$, and 24 weeks post exposure), observation of symptoms (e.g. weekly), and daily self-fever checks. Table 4 shows the laboratory risk levels and indications for PEP.

\section{Conflict of interest}

The authors declare that there are no conflicts of interest.

\section{REFERENCES}

1. Pappas G, Papadimitriou P, Akritidis N, Christou L, Tsianos EV. The new global map of human brucellosis. Lancet Infect Dis. 2006;6(2):91-9.

2. Doganay M. Human brucellosis: importance of brucellosis. Recent Pat Antiinfect Drug Discov. 2013;8(1):2-3.

3. Corbel MJ. Brucellosis in humans and animals. Geneva: World Health Organizaton. 2006. 89p.

4. Dean AS, Crump L, Greter H, Hattendorf J, Schelling E, Zinsstag J. Clinical manifestations of human brucellosis: a systematic review and meta-analysis. PLoS Negl Trop Dis. 2012;6(12):e1929. 
5. Pappas G, Akritidis N, Bosilkovski M, Tsianos E. Brucellosis. N Engl J Med. 2005;352(22):2325-36.

6. Ullmann AJ, Cornely OA, Donnelly JP, Akova M, Arendrup MC, Arikan-Akdagli S, et al. ESCMID* guideline for the diagnosis and management of Candida diseases 2012: developing European guidelines in clinical microbiology and infectious diseases. Clin Microbiol Infect. 2012;18(Suppl 7):1-8.

7. Ariza J, Bosilkovski M, Cascio A, Colmenero JD, Corbel MJ, Falagas ME, et al. Perspectives for the treatment of brucellosis in the 21st century: the Ioannina recommendations. PLoS Med. 2007;4(12):e317.

8. Crawford RP, Huber JD, Adams BS. Epidemiology and surveillance. In: Nielsen K, Duncan JR, editors. Animal Brucellosis: CRC Press; 1990. p. 131-51.

9. Deniz S, Baykam N, Celikbas A, Yilmaz SM, Guzel TC, Dokuzoguz B, et al. Screening Household Members of Acute Brucellosis Cases in Endemic Areas and Risk Factors for Brucellosis. Vector Borne Zoonotic Dis. 2015;15(8):468-72.

10. Griffith J, Sullivan M, Howell J. Laboratory-Acquired Brucellosis. MMWR Morb Mortal Wkly Rep. 2008;57(2):39-42.

11. Dorneles EM, Teixeira-Carvalho A, Araujo MS, Lima GK, MartinsFilho OA, Sriranganathan N, et al. T lymphocytes subsets and cytokine pattern induced by vaccination against bovine brucellosis employing S19 calfhood vaccination and adult RB51 revaccination. Vaccine. 2014;32(46):6034-8.

12. Zhen Q, Lu Y, Yuan X, Qiu Y, Xu J, Li W, et al. Asymptomatic brucellosis infection in humans: implications for diagnosis and prevention. Clin Microbiol Infect. 2013;19(9):E395-7.

13. Dean AS, Crump L, Greter H, Schelling E, Zinsstag J. Global burden of human brucellosis: a systematic review of disease frequency. PLoS Negl Trop Dis. 2012;6(10):e1865.

14. Guven T, Ugurlu K, Ergonul O, Celikbas AK, Gok SE, Comoglu S, et al. Neurobrucellosis: cinical and diagnostic features. Clin Infect Dis. 2013;56(10):1407-12.

15. Pappas G, Bosilkovski M, Akritidis N, Mastora M, Krteva L, Tsianos E. Brucellosis and the respiratory system. Clin Infect Dis. 2003;37(7):e95-9.

16. Vilchez G, Espinoza M, D'Onadio G, Saona P, Gotuzzo E. Brucellosis in pregnancy: clinical aspects and obstetric outcomes. Int J Infect Dis. 2015;38:95-100.
17. Khan LA, Al-Hateeti HS. Brucellosis and thrombocytopenia. Saudi Med J. 2001;22(2):181-2.

18. Rolando I, Olarte L, Vilchez G, Lluncor M, Otero L, Paris M, et al. Ocular manifestations associated with brucellosis: a 26-year experience in Peru. Clin Infect Dis. 2008;46(9):1338-45.

19. Araj GF. Update on laboratory diagnosis of human brucellosis. Int J Antimicrob Agents. 2010;36(Suppl 1):S12-7.

20. Diaz R, Casanova A, Ariza J, Moriyon I. The Rose Bengal Test in human brucellosis: a neglected test for the diagnosis of a neglected disease. PLoS Neg1 Trop Dis. 2011;5(4):e950.

21. Wang Y, Wang Z, Zhang Y, Bai L, Zhao Y, Liu C, et al. Polymerase chain reaction-based assays for the diagnosis of human brucellosis. Ann Clin Microbiol Antimicrob. 2014;13:31.

22. Sohrabi M, Mohabati Mobarez A, Khoramabadi N, Hosseini Doust R, Behmanesh M. Efficient diagnosis and treatment follow-up of human brucellosis by a novel quantitative TaqMan real-time PCR assay: a human clinical survey. J Clin Microbiol. 2014;52(12):4239-43.

23. Bayram Y, Korkoca H, Aypak C, Parlak M, Cikman A, Kilic S, et al. Antimicrobial susceptibilities of Brucella isolates from various clinical specimens. Int J Med Sci. 2011;8(3):198-202.

24. Bodur H, Balaban N, Aksaray S, Yetener V, Akinci E, Colpan A, et al. Biotypes and antimicrobial susceptibilities of Brucella isolates. Scand J Infect Dis. 2003;35(5):337-8.

25. Skalsky K, Yahav D, Bishara J, Pitlik S, Leibovici L, Paul M. Treatment of human brucellosis: systematic review and meta-analysis of randomised controlled trials. BMJ. 2008;336(7646):701-4.

26. Solis Garcia del Pozo J, Solera J. Systematic review and metaanalysis of randomized clinical trials in the treatment of human brucellosis. PLoS One. 2012;7(2):e32090.

27. Alavi SM, Alavi L. Treatment of brucellosis: a systematic review of studies in recent twenty years. Caspian J Intern Med. 2013;4(2):636-41.

28. Yousefi-Nooraie R, Mortaz-Hejri S, Mehrani M, Sadeghipour P. Antibiotics for treating human brucellosis. Cochrane Database Syst Rev. 2012;10:CD007179.

29. Franco MP, Mulder M, Smits HL. Persistence and relapse in brucellosis and need for improved treatment. Trans R Soc Trop Med Hyg. 2007;101(9):854-5. 